\title{
Design and Implementation of Multi-Model Biometric Identification System
}

\author{
Safaa S. Omran \\ Assistant Prof. \\ College of Elec. \& Electronic Techniques, \\ Foundation of Technical Education, \\ Baghdad, Iraq
}

\author{
Maryam A. Salih \\ M.Sc. \\ University of Technology \\ Department of computer engineering \\ Baghdad, Iraq
}

\begin{abstract}
Biometric recognition used to make personal identification based on pattern recognition methods.

This research use fingerprint and iris recognition systems to build an active serial multimodal biometric identification system.

Fingerprint identification algorithm is modified to be modified Delaney triangulation system. In the modified system only the surrounded triangles around each minutia were compared between the input and the stored template.

While in the iris recognition system the segmentation method is modified and the recognition is based on the correlation filter. The recognition method is applied only on the lower part of the iris region that is less effected with noise.
\end{abstract}

The proposed multimodal system gave a high accuracy with error rate equals $(0.9 \%)$.

\section{Keywords}

Multimodal biometric, identification, fingerprint, iris.

\section{INTRODUCTION}

A Biometric recognition system is a combination of hardware and pattern recognition system. It is fundamentally used to distinguish and recognize people as individuals and unique [1].

Biometric recognition system acquire data from an individual, extract silent features set, comperes them against stored features in server database and execute an action based on the result of the comparison algorithm.

Biometric recognition system offers a natural and reliable solution to the problem of identity determination [2].

In the last three decades, unimodel biometric based authentication have being implemented in various applications including computer and cellular phone logins, email, banking, e-commerce transactions and any network login where identity management is critical [3].

Despite the considerable advances in recent years, there are still serious challenges in obtaining reliable authentication through a unimodel biometric system [4].

Unimodel biometric systems (that is biometric systems based on single biometric traits) suffer from several practical problems like noisy sensor data, non-universality, or lake of disconnectedness of biometric traits, unacceptable error rate, and spoof attacks [5].

Multimodal biometric systems which combine multiple biometric samples, or characteristics derived from samples have been developed in order to overcome those problems.
Several studies have been suggested for considering information from multiple biometric traits that can achieve better performance to meet the tight real-world applications requirement [5].

There are number of benefits in using multimodal biometric systems. Just to name few: false acceptance rate and false reject rate will decrease, the authentication system becomes more robust against individual sensor noise, or subsystem failure, and the number of cases where the system where not able to make decision is reduced significantly. The technological environment is also appropriate because of the widespread deployment of minutiae devices [6].

\section{FINGERPRINT RECOGNITION}

\section{SYSTEM}

A fingerprint is the impression of friction ridges, from the surface of a fingertip. The most evident structural characteristics of the fingerprint are a pattern of interleaved ridges and valleys, as shown in Fig. (1).

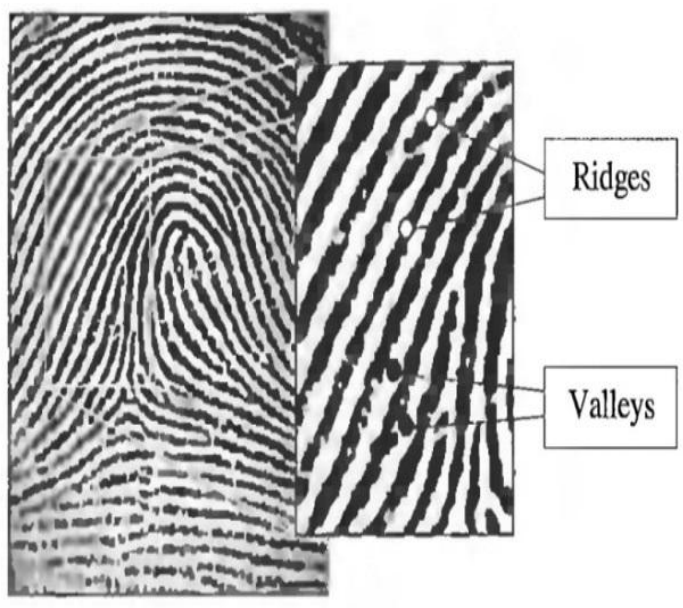

Figure (1) Ridges and valleys on fingerprint image

Injuries such as superficial burns, abrasions, or cuts do not affect the underlying ridge structure, and the original pattern is duplicated in any new skin that grows [7].

At the local level fingerprint there are features called minutiae. Minutiae mean small details. In the context of a fingerprint it refers to the various ways that ridges are discontinued. Where sometimes they terminate called endpoints others bifurcate called bifurcation [8], as 


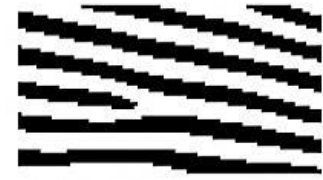

(a) Ridge ending

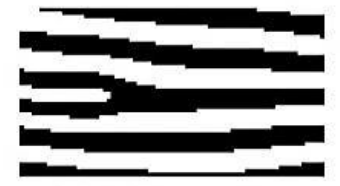

(b) Bifurcation
Figure (2) Example of a ridge ending and a bifurcation

Fingerprint recognition system passes through several sequences of operations in order to get the coded information which is called template. The first operation is fingerprint acquisition to get the fingerprint image. Fingerprint images are enhanced by using some image enhancement techniques, for better system performance. Then feature extraction, these features are stored as template, either to register new user, or to use it to be compared with other fingerprint template, stored in system database [9]. Fig. (3) Represents the functional blocks of the AFRS.

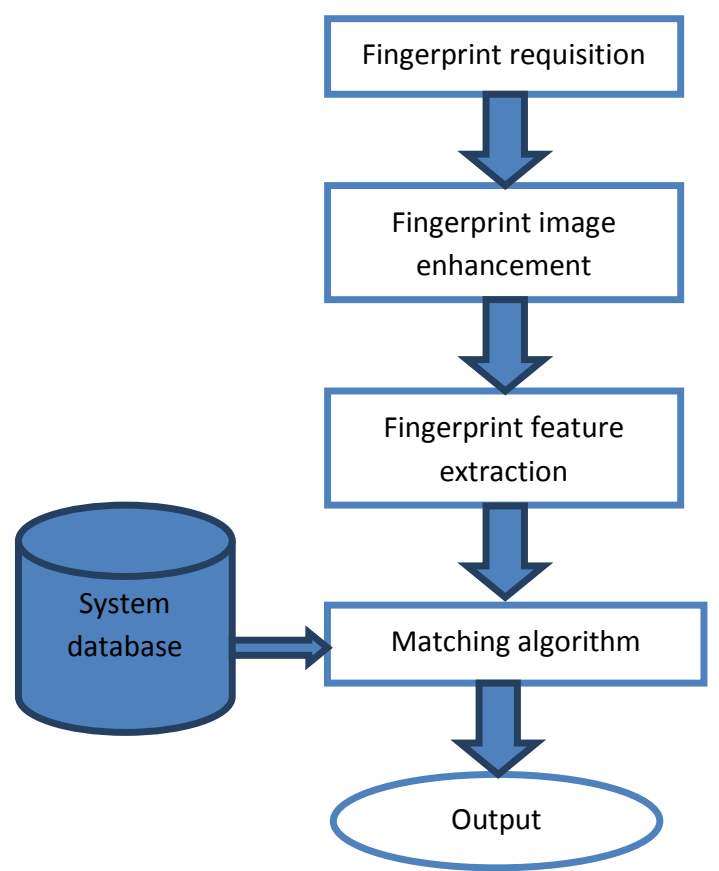

Figure (3) Functional block for AFRS

Fingerprint images are always affected by sweat and dust. Moreover, dry fingers, and scratches on finger, could create some cracked and broken ridges in the fingerprint image; therefore the enhancement phase is a very important task before feature extraction [10].

2D Gabor filter enhance the fingerprint image in both frequency and spatial domains. It has both orientation, and frequency selective properties [11].

2D Gabor filter can be viewed as sinusoidal plane of perpendicular frequency and orientation modulated by Gaussian envelope [12].

An even symmetric Gabor Filter has the following form in spatial domain:

$u(x, y, \theta)=x \cos \theta+y \sin \theta \ldots \ldots \ldots(1)$
$v(x, y, \theta)=-x \sin \theta+y \cos \theta \ldots \ldots \ldots(2)$

$h(x, y, \theta, f)=\frac{1}{2 \pi \sigma_{u} \sigma_{v}} \exp \left\{-\frac{1}{2}\left(\frac{u^{2}}{\sigma_{u}^{2}}+\frac{v^{2}}{\sigma_{v}^{2}}\right)\right\} \cos (f u)$.

Where $\theta$ and $f$ are the orientation and the frequency and $\sigma$ are Gauss envelop constant.

Fingerprint recognition has been studied for many years, and numerous algorithms have been proposed to improve the performance of fingerprint recognition. Among them minutiae-based matching algorithms are the most popular approaches since they are widely believed that minutiae are the most discriminating and reliable features. In minutiae based matching the minutiae are commonly represented by "minutiae triplet" $\mathrm{mi}=(\mathrm{x}, \mathrm{y}, \theta)$, Where $(\mathrm{x}, \mathrm{y})$ is the index of the minutiae at the Cartesian-axis, and $\theta$ is the orientation angle for the minutiae points. For matching process a matching minutia pairs is counted.

Generally, there are problems in fingerprint minutiae triplet matching algorithm a too small overlap, loss of genuine minutiae and pseudo minutiae extraction localization deviation of minutiae.

A good improvement to the above algorithm is fingerprint matching algorithm using the minutiae triangles, to cope with the strong deformation of fingerprint image. By connect the minutiae points of single fingerprint to form Triangulation Net (TN) called Delaunay triangulation (DT) map based on the algorithm used to form TN.

For matching process after creating minutiae triplets and forming $\mathrm{TN}$, each triangle vertices is represented in the form $(\alpha, l, \theta), \alpha$ is the vertices angle, $l$ is the edge length, and $\theta$ is the vertices orientation. $\mathrm{T}\left(\alpha_{\mathrm{ti}}, \mathrm{l}_{\mathrm{ti}}, \theta_{\mathrm{ti}}\right)$ and $\mathrm{I}\left(\alpha_{\mathrm{ii}}, \mathrm{l}_{\mathrm{ii}}, \theta_{\mathrm{ii}}\right)$ from the template and input TN net, respectively. Two triangles in the input and template TN are creating triangle pair only when the following equations are satisfied:

$\left|\mathrm{l}_{\mathrm{ti}}-\mathrm{l}_{\mathrm{ii}}\right|<\mathrm{T}_{1}$,

$\left|\theta_{\mathrm{ti}}-\theta_{\mathrm{ii}}\right|<\mathrm{T}_{2}$

$\left|\alpha_{\mathrm{ti}}-\alpha_{\mathrm{ii}}\right|<\mathrm{T}_{3}$,

where $\mathrm{i}=1,2,3$ are the triangle vertics

Where T1, T2, T3 are the threshold of the edge length, vertices orientation and vertices angle [13]. Fig. (4) shows a $\mathrm{TN}$ and its local features.

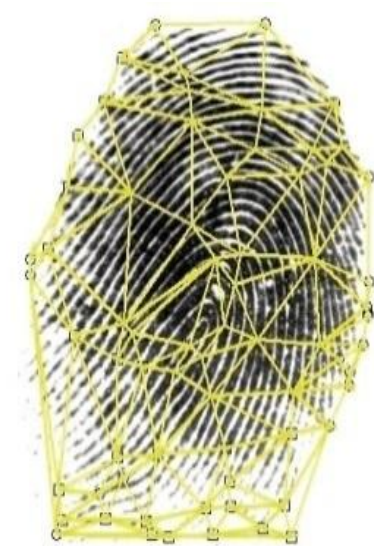

(a)Delaunay Triangulation map

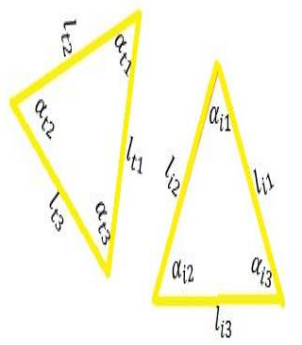

(b)local features of two triangles for input fingerprint and template fingerprint

Figure (4) TN structure 


\section{RIS RECOGNITION SYSTEM}

The human iris is an annular part between pupil and sclera. The uniqueness and stability of the iris features make it very attractive to be used as biometric identification for the individuals. Fig. (5) Shows a block diagram for Iris recognition system.

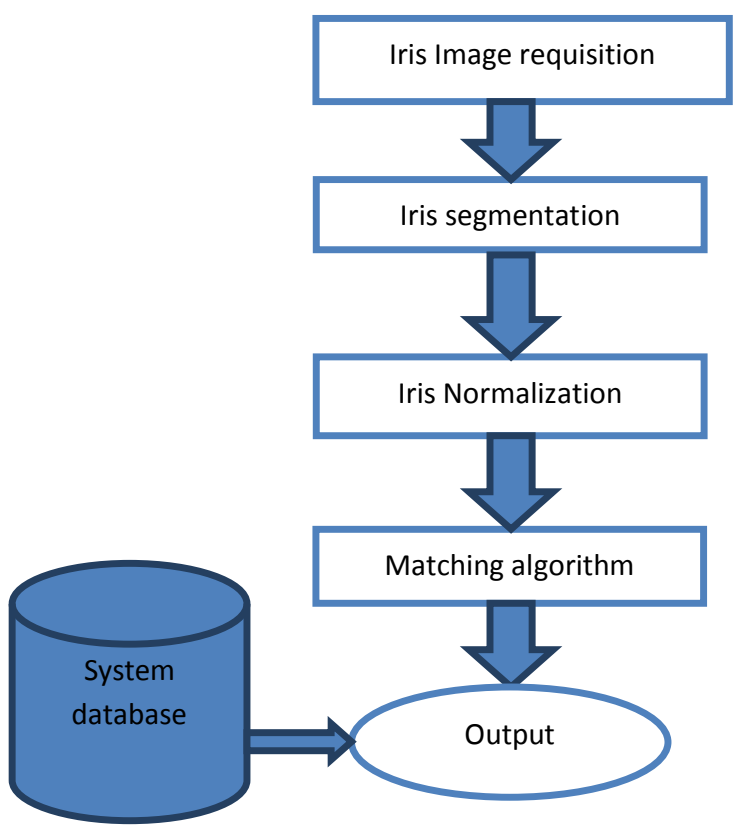

Figure (5) Functional block for iris recognition system

First the eye image is acquired using specialized camera, and then a template is created for the acquired iris image. The iris template contains an objective mathematical representation of the unique information stored in the iris. This template is compared with other template stored in the database until either a matching template is found and subject is defined or no match is found and subject remains undefined [14].

Iris localization and segmentation is the process of finding the inner and the outer boundary of the iris area.

Iris localization and segmentation is quiet challenging because of all kinds of drawbacks of iris image like (motion blurred, glasses, eyelid, eyelashes, and reflections ...etc.) [15].

Once the segmentation module estimates the iris boundary, the normalization module transforms the iris texture from Cartesian to polar coordinate. The process is often called iris unwarping which yields a rectangular entity that used for subsequent processing [16]. As shown in Fig.(6)

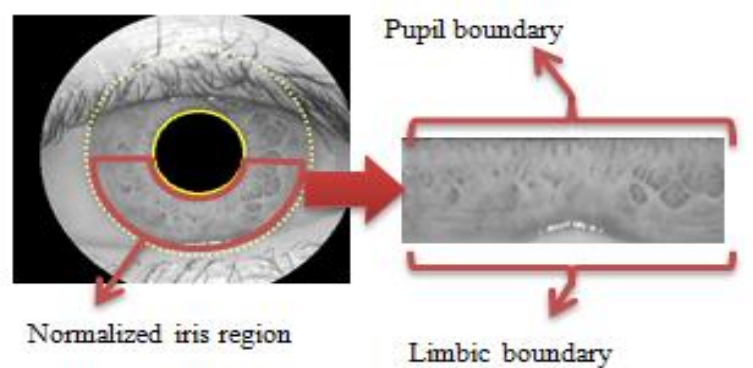

Figure (6) Normalization Process
Different efficient algorithms use phase-based image matching techniques. Wildes[17], was the first to propose matching iris by correlation filter by applying Lablasian filters, and fisher analysis. Since then several works have explored correlation measures.

Other researchers proposed a new phase correlation technique introduced based method of iris in frequency domain using Fourier phase information [18].

The correlation filters spatial frequency domain array that specifically designed to recognize a particular pattern class represented as set of reference patterns. A given query pattern is matched against this template by performing a crosscorrelation. To make this efficient, cross-correlation is performed in the frequency domain taking advantage of DFT. Fig. (7) Describe cross correlation iris recognition system [19].

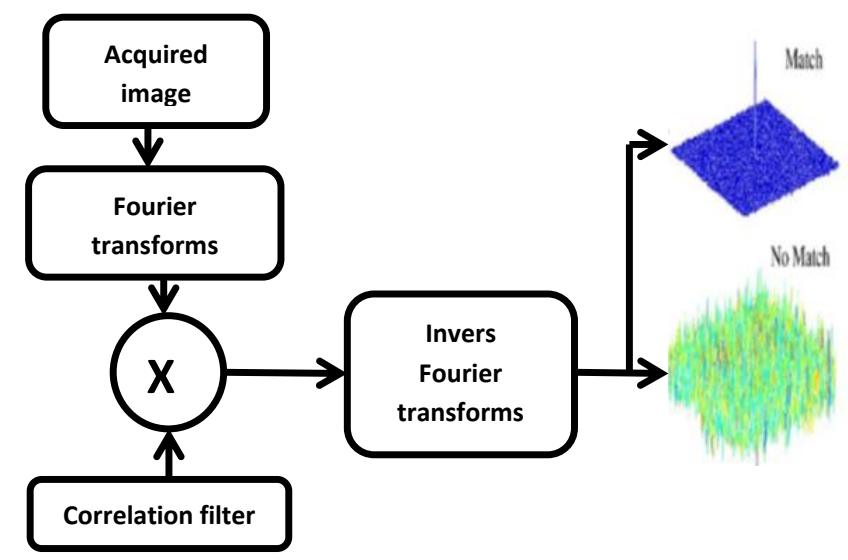

Figure (7) Iris recognition using cross-correlation

Two $N_{1} \times N_{2}$ images, $f\left(n_{1}, n_{2}\right)$ and $f\left(n_{1}, n_{2}\right)$, where assumed that index ranges are $n_{1}=-M_{1} \ldots M_{1}\left(M_{1}>0\right)$ and $n_{2}=$ $-M_{2} \ldots M_{2}\left(M_{2}>0\right)$, and hence $N_{1}=2 M_{1}+1$ and $N_{2}=$ $2 M_{2}+1$. Let $\mathrm{F}\left(\mathrm{k}_{1}, \mathrm{k}_{2}\right)$ and $\mathrm{G}\left(\mathrm{k}_{1}, \mathrm{k}_{2}\right)$ denote $2 \mathrm{D}$ DFT of two images, $F\left(k_{1}, k_{2}\right)$ is given by

$\mathrm{F}(\mathrm{k} 1, \mathrm{k} 2)=\sum_{n 1=-M_{1}}^{M_{1}} \sum_{n 2=-M_{2}}^{M_{2}} f\left(n_{1}, n_{2}\right) W_{N_{1}}^{k_{1}} W_{N_{2}}^{k_{2}} \ldots \ldots$

Where $k_{1}=-\mathrm{M}_{1} \ldots . \mathrm{M}_{1}, \quad k_{2}=-\mathrm{M}_{2} \ldots . \mathrm{M}_{2}, \quad W_{N 1}=e^{-j \frac{2 \pi}{N 1}}$, and $W_{N 2}=e^{-j \frac{2 \pi}{N 2}} . . \mathrm{G}\left(\mathrm{k}_{1}, \mathrm{k}_{2}\right)$ is defined in the same way. The crossphase spectrum $R_{F G}\left(k_{1}, k_{2}\right)$ between $\mathrm{F}\left(\mathrm{k}_{1}, \mathrm{k}_{2}\right)$ and $\mathrm{G}\left(\mathrm{k}_{1}, \mathrm{k}_{2}\right)$ is given by

$R_{F G}\left(k_{1}, k_{2}\right)=\frac{\mathrm{F}(\mathrm{k} 1, \mathrm{k} 2) \overline{\mathrm{G}(\mathrm{k} 1, \mathrm{k} 2)}}{|\mathrm{F}(\mathrm{k} 1, \mathrm{k} 2) \overline{\mathrm{G}(\mathrm{k} 1, \mathrm{k} 2)}|} \ldots \ldots \ldots$

Where $\overline{\mathrm{G}(\mathrm{k} 1, \mathrm{k} 2)}$ is the complex conjugate of $\mathrm{G}(\mathrm{k} 1, \mathrm{k} 2)$. The height of the peak can be used as a similarity measure for image matching [20].

To quantify the degree of sharpness of the correlation peak, Peak-To-Correlation-Energy (PCE) for the correlation plan is measured to define the similarity using the following equation [21]:

$P C E=\frac{\text { maxpeak }- \text { mean }(\text { correlation } \text { plan })}{\text { standerd diviation }(\text { correlation } \text { plan })} \ldots$ 


\section{PROPOSED MULTIMODAL RECOGNITION SYSTEM}

This research propos building multimodal biometric recognition system based on fingerprint and iris trait, due to their highly accurate response, and they have short recognition time when they work in uni-model.

First iris recognition system is used in order to reduce the number of comparisons in the fingerprint recognition system. Since its recognition time is much less than that of the fingerprint recognition system.

The input iris template is compared with all templates stored in the iris database. If the matching score between the input template and a stored template is above the threshold, then a pointer to the stored template is stored in candidate list. This makes iris recognition system selects a number of candidates to look at by the fingerprint recognition system for the final decision.

The final output decision is based on both systems which make it more reliable and less error. The claimed person can be recognized if and only if both systems recognize that candidate.

The proposed system improves the performance of the iris and finger print recognition systems by improving the segmentation process in the iris recognition phase and the matching method in the finger print phase.

This modification improves both iris and fingerprint systems as individual and that improves the overall recognition rate for the whole multi model system, Fig. (8) Shows a block diagram for the multi model system.

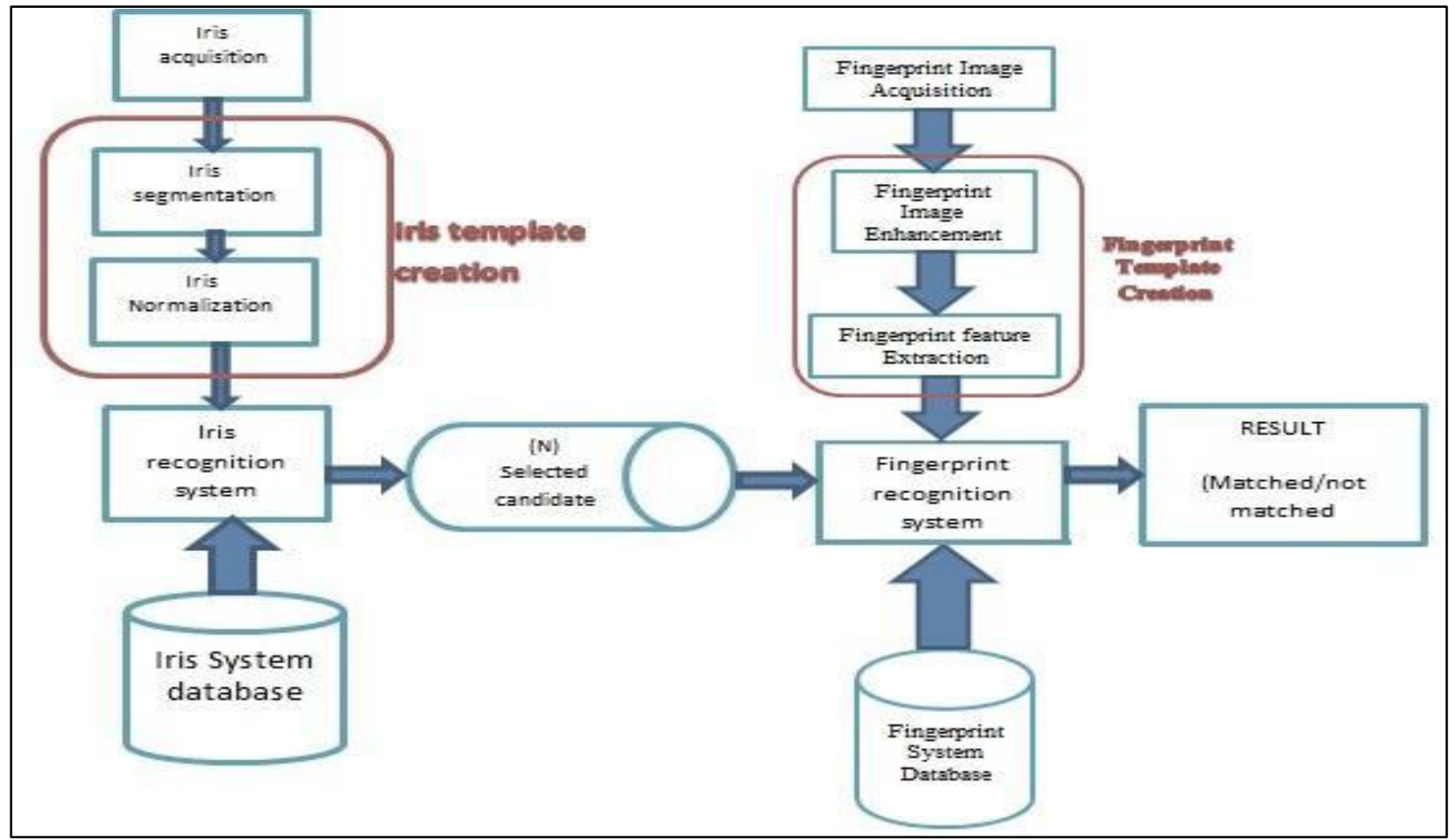

Figure (8) User Identification block diagram

\section{MODIFIED HISTOGRAM BASED IRIS SEGMENTATION}

The grey scale iris image is divided into different regions with different intensity level this regions are: pupil, sclera, eyelids, eyelash, and skin. Base on that fact the iris area will be segmented from the other part of the eye image.

The proposed method is working in two stages: (a) pupil localization, (b) iris segmentation.

\subsection{Pupil localization:-}

A binary image is created using image histogram. The binary image represents the darkest regions in the eye image.

Sometime the darkest region in the binary image includes eye lashes. By connect the binary image pixels into groups, and labeling each group with different color.

Standard deviation of the coordinates $(x, y)$ corresponding to each group is computed. The group with the less standard deviation is considered as the pupil region. The follow operation of the pupil localization is shown in Fig. (9).

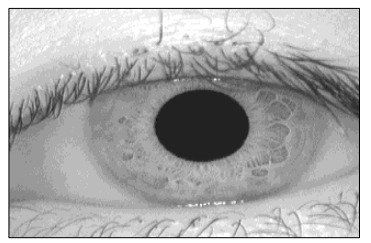

(a) The original image

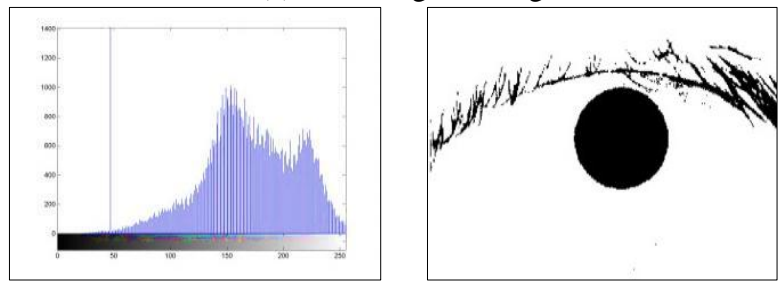

(b) Image histogram

(c) Binary image 


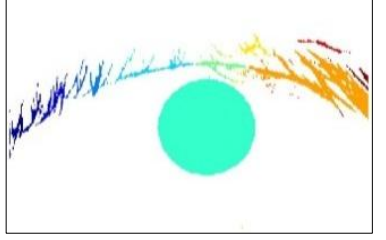

(d) Binary image labeled groups

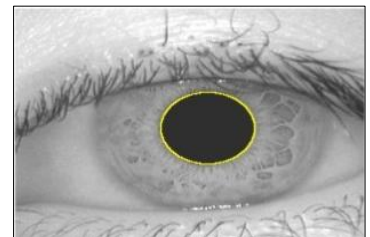

(e) Final pupil allocation
Figure (9) pupil localization process

\subsection{Segmentation}

The iris localization is started by segments the region around the pupil with circular mask. As shown in Fig. (10), this will reduce the noise; the pupillary area is set to zero.

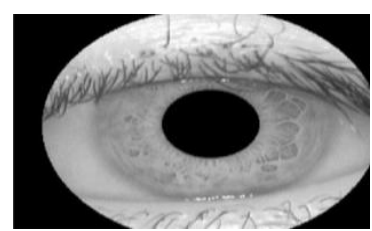

Figure (10)circular mask

To enhance the boundary between the iris and the sclera, the contrast for the eye image has to be enhanced by grouping different neighbors gray scale values into one cluster to get sharper edge between the iris and the sclera, as shown in Fig. (11).

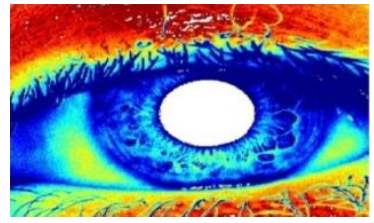

Figure (11) image color clustering

A navigated line inside the circular mask will move at the bottom half of the iris image to collect the region information as shown in Fig. (12).

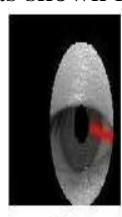

(1)

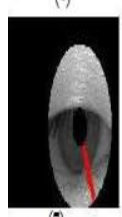

(7)

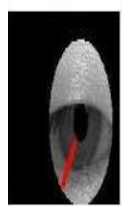

(13)

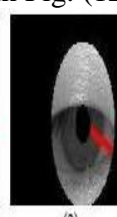

(2)

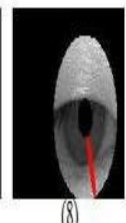

(1)

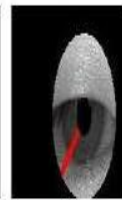

(14)

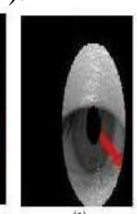

(3)

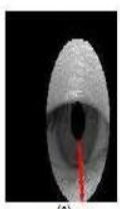

(9)

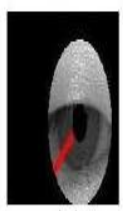

(15)

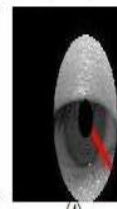

(4)

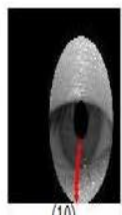

(10)

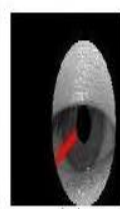

(16)

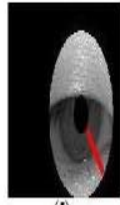

(5)

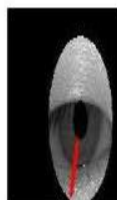

(11)

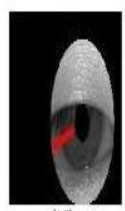

(17)

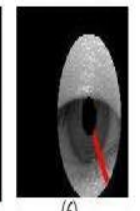

(6)

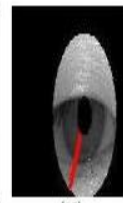

(12)

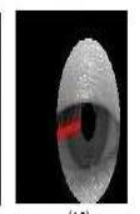

(18)

The observed information is used to track boundary point that can be used to obtain the iris outer boundary as shown in Fig. (13)

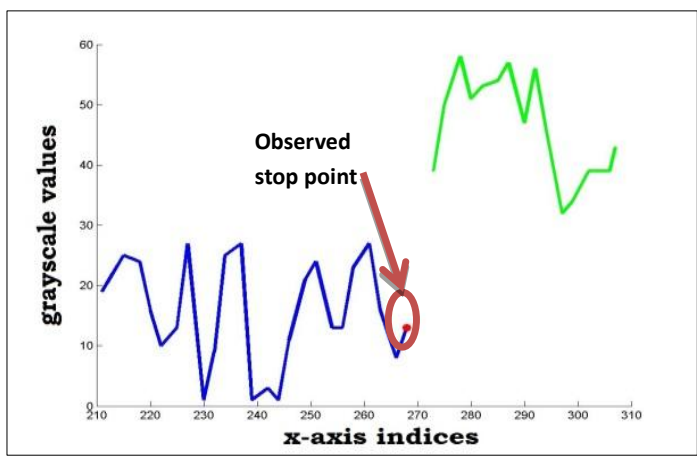

(a) Observed line information

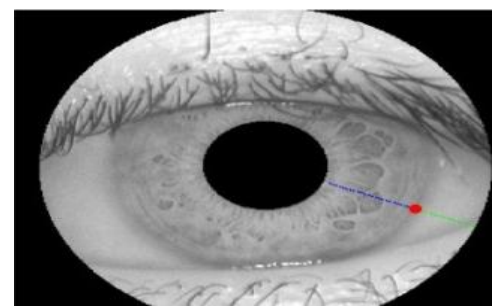

(b) finding stop point

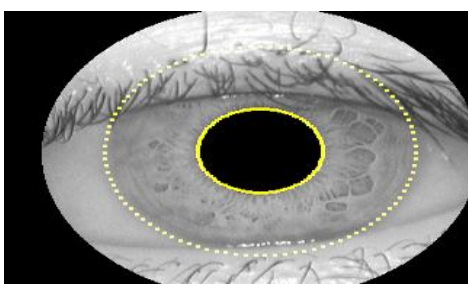

(C) final iris allocation

Figure (13) iris segmentation process

\section{THE MODIFIED FINGERPRINT RECOGNITION PROCESS}

A modified Delaunay triangulation matching algorithm is used for matching between two fingerprint images. This method combines traditional minutiae matching algorithm and Delaunay triangulation.

The modified Delaunay triangulation start by creating a Delaunay triangulation network, finding the matching minutia triplet pairs using the traditional way, then remove the false minutiae pairs using the surrounded angels that was generated using Delaunay network. This method adds other property to the minutia which is the surrounded angles that is generated by Delaunay triangulation which gives more certainty in finding minutia pairs.

The following steps describe the matching process:

1. Extract the minutia in both compared fingerprint images.

2. Implement triangulation network between the both compared fingerprint images.

Figure (12) navigating line movement 
3. Finding all three edge lengths of all triangles in the whole triangulation network in both compared fingerprint images.

4. Finding the inner angles for all triangles in the triangulation network.

5. Finding the minutiae pairs between both compared fingerprint images by using the traditional method.

6. Finding the surrounding angles for each minutia pair.

7. Match the surrounded angles between each two minutia pairs if they are equal consider it as certain minutia pair.

8. Count the certain minutia pares if the number above threshold then the two templates are matched.

Fig. (14) The output after finding certain minutia pairs.
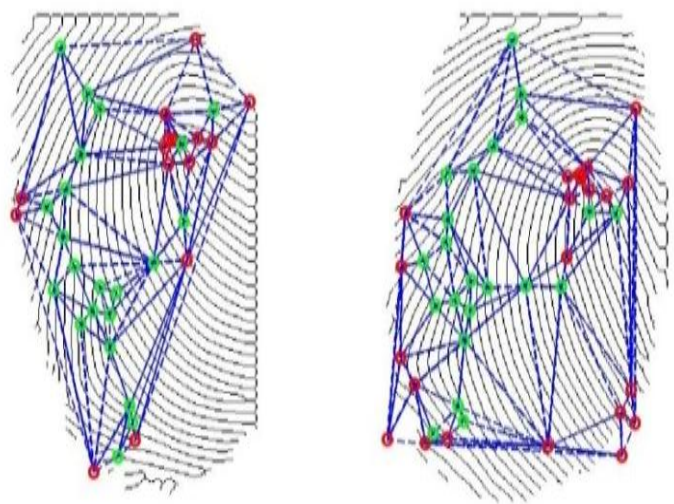

(a) two samples from the same fingerprint
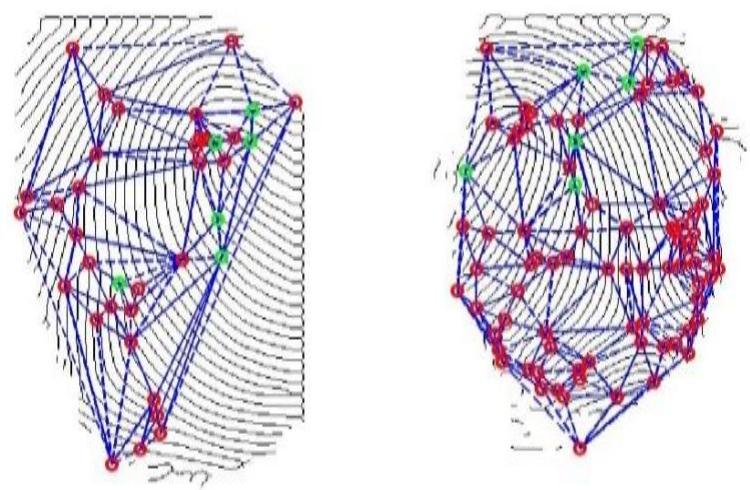

(b) two samples from the different fingerprint

Figure (14) Two fingerprint after applying modified Delaunay triangulation net fingerprint, green marks are certain minutiae pairs and red marks are unpaired minutiae

\section{SYSTEM RESULT}

Personal identification based on biometric traits is a challenge research issue, due to noise that affects the biometric traits and the intra-class variation problem. Therefor most researchers seek for an identification system with lower error rate. Every biometric identification system has two types of errors:-

1. False Matching Rate (FMR): is the number of samples that are considered to be matched even if they are from different person.
2. False Not Matching Rate (FNMR): is the number of samples that are considered as not matching even if they are from the same person.

FMR and FNMR are plotted as a function of threshold (th) the point where FMR (th) and FNMR (th) intersect is called Equal Error Rate (EER). The lower the EER is the better system performance.

To evaluate the performance of the proposed system, two public biometric databases were merged, (MMU iris database and FVC2004 database), to create the multimodal database. The mutual independence assumption of the biometric traits allows to randomly pair the users from the two sets collected using different sensors and over different time periods.

The following figures are the error rate plot figures that evaluate iris and fingerprint uni-model recognition system and the multimodal implemented using iris and fingerprint traits.

The error rate plot shown in Fig (15) represents the performance of minutia based fingerprint matching system. As it is seen in the figure, the EER equals $(11,11 \%)$ at (threshold equals 14.24).

While Fig. (16) represents the performance of the fingerprint matching system after applying the modified Delaunay triangulation matching method with ERR equals $(8.333 \%)$ at (threshold equals 7.466).

As it seem that EER decreases by $2.777 \%$.

Fig. (17) and figure (18) represent the performance of correlation filter based iris matching system. In Fig. (17) the matching method used the whole iris area. The EER was $(15.35 \%)$ at (threshold equals 4.937$)$.

While in Fig. (18) the matching system used only the lower half of the iris area. The results of EER equals (12.32\%) at (threshold equals 5.894). It is clear that using only the lower half of iris area gives better performance and the EER decreases by $3.03 \%$.

Fig. (19) shows that combining fingerprint and iris recognition systems to build multimodal system (where input template is accepted if only both systems are accepted its input template), decrease the error rate significantly, where the final EER equals $(0.9259 \%)$ at threshold equals (6.581).

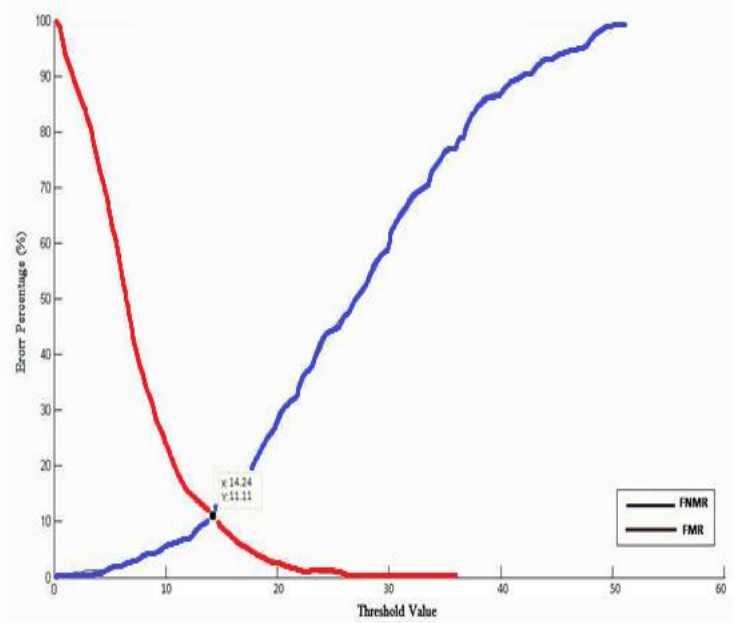

Figure (15) Error rate plot for minutia based fingerprint matching system 


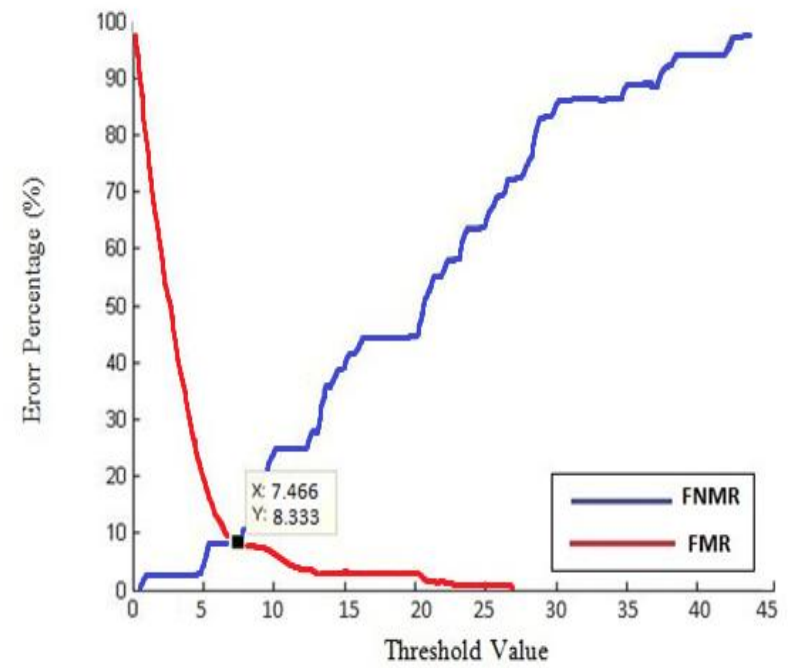

Figure (16) Error rate plot for modified Delaunay triangulation based fingerprint matching system

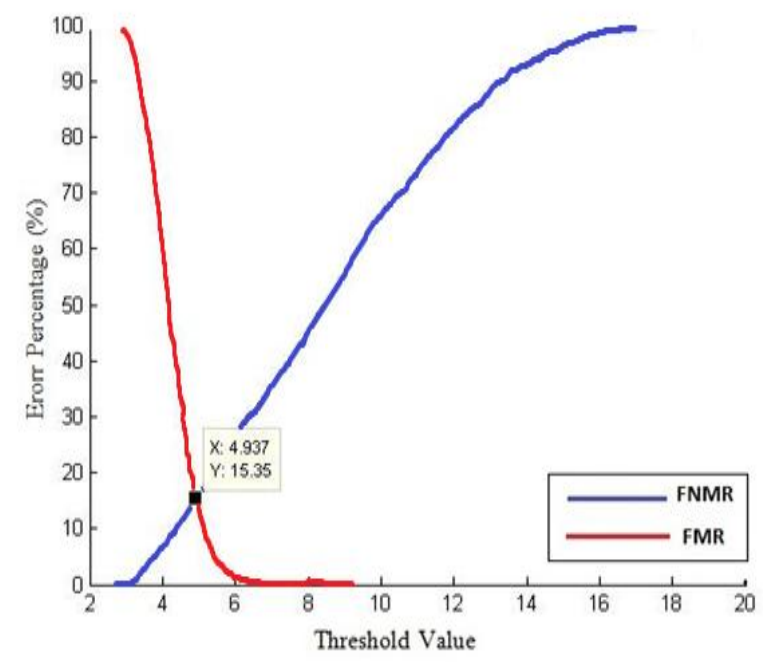

Figure (17) Error rate plot for correlation filter based iris matching system using the whole iris area

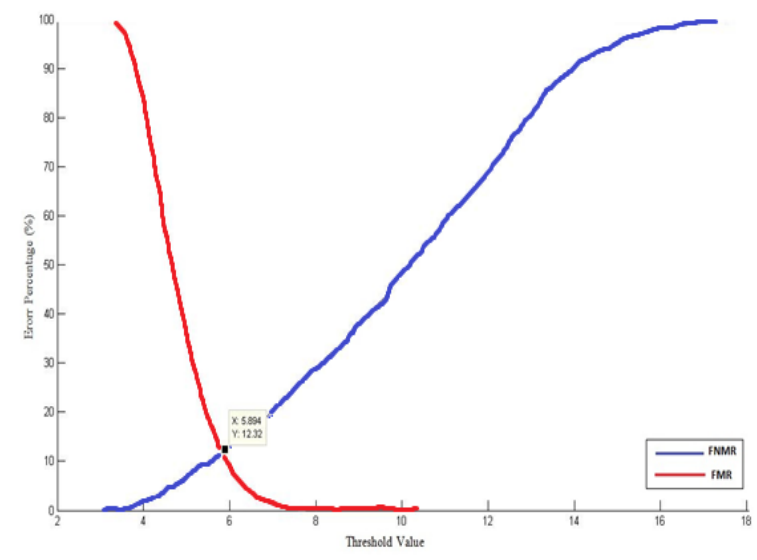

Figure (18) Error rate plot for correlation filter based iris matching system using the lower half of the iris area

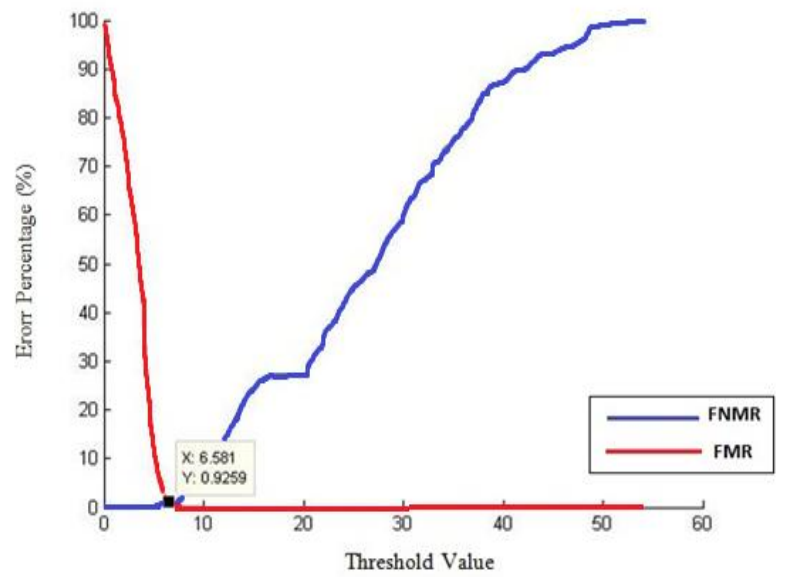

Figure (19) Error rate plot for multimodal biometric recognition system

\section{CONCLUSION}

This research discusses the use of iris and fingerprint traits to build multimodal biometric recognition system.

The performance of the fingerprint recognition system is increased by modifying the matching algorithm instead of searching the whole Delaunay triangulation network. The modified method only searches the triangles around each paired minutia.

In iris recognition system, the enhanced iris segmentation method increases the performance for the whole system. The segmentation process is a demand part of the iris recognition system where the whole system performance depends on it. Using fast recognition method decreases the response of the multimodal recognition system. And instead of using the whole iris area, only the lower part used that would help to decrease the noise caused by eyelashes that mostly affect the upper part of the iris area.

All the modifications on the fingerprint and the iris recognition system give a satisfying result and the research goal was achieved. By combining strong biometric traits the multimodal biometric system performance gave very low error rate.

\section{REFERENCES}

[1] T. Dunstone, and N. Yager, "Biometric System and Data Analysis Design, Evaluation, and Data Mining", Springer, 2009.

[2] K. Nandakumar," Multibiometric Systems: Fusion Strategies And Template Security", Department Of Computer Science And Engineering, Ph.D. Thesis, 2008 .

[3] W. Almayyan, "Performance Analysis of Multimodal Biometric Fusion", De Montfort University, M.Sc. Thesis, 2012.

[4] F. Alsaade, "Score-Level Fusion for Multimodal Biometrics", University Of Hertfordshire, M.Sc. Thesis, 2008. 
[5] K. Sentosa, " Performance Evaluation of Score Level Fusion in Multimodal Biometric Systems", Department Of Computer Science And Information Engineering National Taiwan University Of Science And Technology, M.Sc. Thesis, 2007.

[6] J. Aguilar, "adoptive Fusion Scheme for multimodal biometric Authentication ", Univirsity Of Politecnica De Madrid, Ph.D. Thesis, 2006.

[7] D. Maltoni, D. Maio, A. Jain, and S. Prabhakar, "Handbook of Fingerprint Recognition", SpringerVerlag New York, Inc., 2003.

[8] N. Ratha and R. Bolle," Automatic Fingerprint Recognition Systems", Springer,2004.

[9] V. Areekul, U. Watchareeruetai, K. Suppasriwasuseth, and S. Tantaratana," Separable Gabor Filter Realization for Fast Fingerprint Enhancement", IEEE Image Processing, ICIP2005 International Conference, Genoa, Italy, 2005.

[10] K. Arora, and P. Garg, "A Quantitative Survey Of Various Fingerprint Enhancement Techniques", International Journal Of Computer Applications Vol. 28 No.5, 2011.

[11] K. Arora, and P. Garg," A Quantitative Survey Of Various Fingerprint Enhancement Techniques", International Journal Of Computer Applications, Vol. 28, No.5, 2011.

[12] N. Liu, Y. Yin, H. Zhang," A Fingerprint Matching Algorithm Based On Delaunay Triangulation Net", IEEE Computer and Information Technology, 2005. CIT2005. The Fifth International Conference, Shanghai, 2005.
[13] H. Gite, C. Mahender," Iris Code Generation And Recognition", International Journal Of Machine Intelligence (IJMI),Vol.26, No. 11, 2011.

[14] Q. Wang, X. Zhang, M. Li, X. Dong, Q. Zhou, and Y. Yin," Adaboost And Multi-Orientation 2d Gabor-Based Noisy Iris Recognition", Pattern Recognition Letters Vol. 33, No.8, 2012.

[15] S. Nithyanandam, K. Gayathri , and P. Priyadarshini," A New IRIS Normalization Process For Recognition System With Cryptographic Techniques", IJCSI International Journal Of Computer Science, Vol. 8, Issue 4, No 1, 2011.

[16] R. Farouk," Iris Recognition Based On Elastic Graph Matching And Gabor Wavelets", Computer Vision And Image Understanding, Vol. 115, No. 12, 2011.

[17] J. Thornton, and M. Savvides, B. Kumar, "A Bayesian Approach to deformed Pattern Matching of Iris Image", IEEE Pattern Analysis And Machine Intelligence, Vol. 29, No. 4, 2007

[18] K. Miyazawa, K. Ito, T. Aoki, K. Kobayashi, and H. Nakaima, "A Phase-Based Iris Recognition Algorithm", Springer-Verlag Berlin Heidelberg, 2005

[19] V. Boddeti, and V. Kumar, "Extended Depth of Field Iris Recognition Using Unrestored Wavefront Coded Imagery", IEEE transactions on systems, man, and cybernetics part A: systems and humans, Vol. 40, No. 3, 2010. 